This is a self-archived version of an original article. This version may differ from the original in pagination and typographic details.

Author(s): PHENIX Collaboration

Title: Creation of quark-gluon plasma droplets with three distinct geometries

Year: 2019

Version: Accepted version (Final draft)

Copyright: @ 2018, Springer Nature

Rights: In Copyright

Rights url: http://rightsstatements.org/page/lnC/1.0/?language=en

Please cite the original version:

PHENIX Collaboration. (2019). Creation of quark-gluon plasma droplets with three distinct geometries. Nature Physics, 15(3), 214-220. https://doi.org/10.1038/s41567-018-0360-0 


\title{
Creation of quark-gluon plasma droplets with three distinct geometries
}

\author{
C. Aidala, B. Azmoun
}

To be published in "NATURE PHYSICS"

March 2019

Physics Department

\section{Brookhaven National Laboratory}

\section{U.S. Department of Energy}

USDOE Office of Science (SC), Nuclear Physics (NP) (SC-26)

\footnotetext{
Notice: This manuscript has been authored by employees of Brookhaven Science Associates, LLC under Contract No. DE-SC0012704 with the U.S. Department of Energy. The publisher by accepting the manuscript for publication acknowledges that the United States Government retains a non-exclusive, paid-up, irrevocable, world-wide license to publish or reproduce the published form of this manuscript, or allow others to do so, for United States Government purposes.
} 


\section{DISCLAIMER}

This report was prepared as an account of work sponsored by an agency of the United States Government. Neither the United States Government nor any agency thereof, nor any of their employees, nor any of their contractors, subcontractors, or their employees, makes any warranty, express or implied, or assumes any legal liability or responsibility for the accuracy, completeness, or any third party's use or the results of such use of any information, apparatus, product, or process disclosed, or represents that its use would not infringe privately owned rights. Reference herein to any specific commercial product, process, or service by trade name, trademark, manufacturer, or otherwise, does not necessarily constitute or imply its endorsement, recommendation, or favoring by the United States Government or any agency thereof or its contractors or subcontractors. The views and opinions of authors expressed herein do not necessarily state or reflect those of the United States Government or any agency thereof. 


\title{
Creation of quark-gluon plasma droplets with three distinct geometries
}

\author{
PHENIX Collaboration*
}

\begin{abstract}
Experimental studies of the collisions of heavy nuclei at relativistic energies have established the properties of the quarkgluon plasma (QGP), a state of hot, dense nuclear matter in which quarks and gluons are not bound into hadrons ${ }^{1-4}$. In this state, matter behaves as a nearly inviscid fluid ${ }^{5}$ that efficiently translates initial spatial anisotropies into correlated momentum anisotropies among the particles produced, creating a common velocity field pattern known as collective flow. In recent years, comparable momentum anisotropies have been measured in small-system proton-proton $(p+p)$ and proton-nucleus $(p+A)$ collisions, despite expectations that the volume and lifetime of the medium produced would be too small to form a QGP. Here we report on the observation of elliptic and triangular flow patterns of charged particles produced in proton-gold $(p+A u)$, deuteron-gold $(d+A u)$ and helium-gold ( ${ }^{3} \mathrm{He}+\mathrm{Au}$ ) collisions at a nucleon-nucleon centreof-mass energy $\sqrt{s_{\mathrm{NN}}}=200 \mathrm{GeV}$. The unique combination of three distinct initial geometries and two flow patterns provides unprecedented model discrimination. Hydrodynamical models, which include the formation of a short-lived QGP droplet, provide the best simultaneous description of these measurements.
\end{abstract}

Experiments at the Relativistic Heavy Ion Collider (RHIC) and the Large Hadron Collider (LHC) explore emergent phenomena in quantum chromodynamics, most notably the near-perfect fluidity of the QGP. To quantify this behaviour, the azimuthal distribution of each event's final-state particles, $\frac{\mathrm{d} N}{\mathrm{~d} \phi}$, is decomposed into a Fourier series

$$
\frac{\mathrm{d} N}{\mathrm{~d} \phi} \propto 1+\sum_{n} 2 v_{n}\left(p_{\mathrm{T}}\right) \cos \left(n\left(\phi-\psi_{n}\right)\right)
$$

where $p_{\mathrm{T}}$ and $\phi$ are the transverse momentum and the azimuthal angle of a particle relative to the beam direction, respectively, and $\psi_{n}$ is the orientation of the $n$th order symmetry plane of the produced particles. The second $\left(v_{2}\right)$ and third $\left(v_{3}\right)$ Fourier coefficients represent the amplitude of elliptic and triangular flow, respectively. A multitude of measurements of the Fourier coefficients, utilizing a variety of techniques, have been well described by hydrodynamical models, thereby establishing the fluid nature of the QGP in largeion collisions ${ }^{5}$.

The LHC experiments were first to observe similar features in small-system collisions ${ }^{6-9}$, followed closely by reanalysis of previously recorded $\mathrm{d}+\mathrm{Au}$ data from the $\mathrm{RHIC}^{10,11}$. These unexpected results highlighted the need to explore whether these smallest hadronic systems still form QGP. Alternatively, a number of physics mechanisms that do not involve QGP formation have been proposed, including those that attribute final-state momentum anisotropy to momentum correlations generated at the earliest stages of the collision, hence referred to as initial-state momentum correlation models (see refs ${ }^{12,13}$ for recent reviews).

A projectile geometry scan utilizing the unique capabilities of the RHIC was proposed in ref. ${ }^{14}$ to discriminate between hydrodynamical models that couple to the initial geometry and initial-state momentum correlation models that do not. Varying the collision system from $\mathrm{p}+\mathrm{Au}$, to $\mathrm{d}+\mathrm{Au}$, to ${ }^{3} \mathrm{He}+\mathrm{Au}$ changes the initial geometry from dominantly circular, to elliptical, to triangular configurations, respectively, as characterized by the second- and third-order spatial eccentricities, which correspond to ellipticity and triangularity, respectively. The $n$th order spatial eccentricity of the system, $\varepsilon_{n}$, typically determined from a Monte Carlo (MC) Glauber model of nucleon-nucleon interactions (see for example ref. ${ }^{15}$ ), can be defined as

$$
\varepsilon_{n}=\frac{\sqrt{\left\langle r^{n} \cos (n \phi)\right\rangle^{2}+\left\langle r^{n} \sin (n \phi)\right\rangle^{2}}}{\left\langle r^{n}\right\rangle}
$$

where $r$ and $\phi$ are the polar coordinates of participating nucleons ${ }^{16}$. The eccentricity fluctuates event-by-event and is generally dependent on the impact parameter of the collision and the number of participating nucleons. The mean $\varepsilon_{2}$ and $\varepsilon_{3}$ values for small impact parameter $\mathrm{p} / \mathrm{d} /{ }^{3} \mathrm{He}+\mathrm{Au}$ collisions are shown in Fig. 1a. The $\varepsilon_{2}$ and $\varepsilon_{3}$ values in $\mathrm{d}+\mathrm{Au}$ and ${ }^{3} \mathrm{He}+\mathrm{Au}$ are driven almost entirely by the intrinsic geometry of the deuteron and ${ }^{3} \mathrm{He}$, while the values in $\mathrm{p}+\mathrm{Au}$ collisions are driven by fluctuations in the configuration of struck nucleons in the Au nucleus, as the proton itself is, on average, circular.

Hydrodynamical models begin with an initial spatial energydensity distribution with a given temperature that evolves in time following the laws of relativistic viscous hydrodynamics using an equation of state determined from lattice quantum chromodynamics ${ }^{17}$. Examples of this evolution are shown for $\mathrm{p} / \mathrm{d} /{ }^{3} \mathrm{He}+\mathrm{Au}$ collisions in Fig. 1b using the hydrodynamical model SONIC ${ }^{18}$. The first panel of each row shows the temperature profile at time $t=1.0 \mathrm{fm} c^{-1}$ for typical head-on $\mathrm{p}+\mathrm{Au}, \mathrm{d}+\mathrm{Au}$ and ${ }^{3} \mathrm{He}+\mathrm{Au}$ collisions, where $c$ is the speed of light in vacuum. The following three panels show snapshots of the temperature evolution at three successive time points. The initial spatial distribution also sets the pressure gradient field, which translates into a velocity field, which in turn determines the azimuthal momentum distribution of produced particles. The relative magnitude and direction of the velocity is represented in the figure by arrows. At the final time point, $t=4.5 \mathrm{fm} c^{-1}$, the mostly circular (top), elliptical (middle) and triangular (bottom) initial spatial eccentricities have been translated into dominantly radial, elliptic and triangular flow, respectively.

Given these different initial geometries, as characterized by the $\varepsilon_{2}$ and $\varepsilon_{3}$ values shown in Fig. 1a, hydrodynamical models provide a 

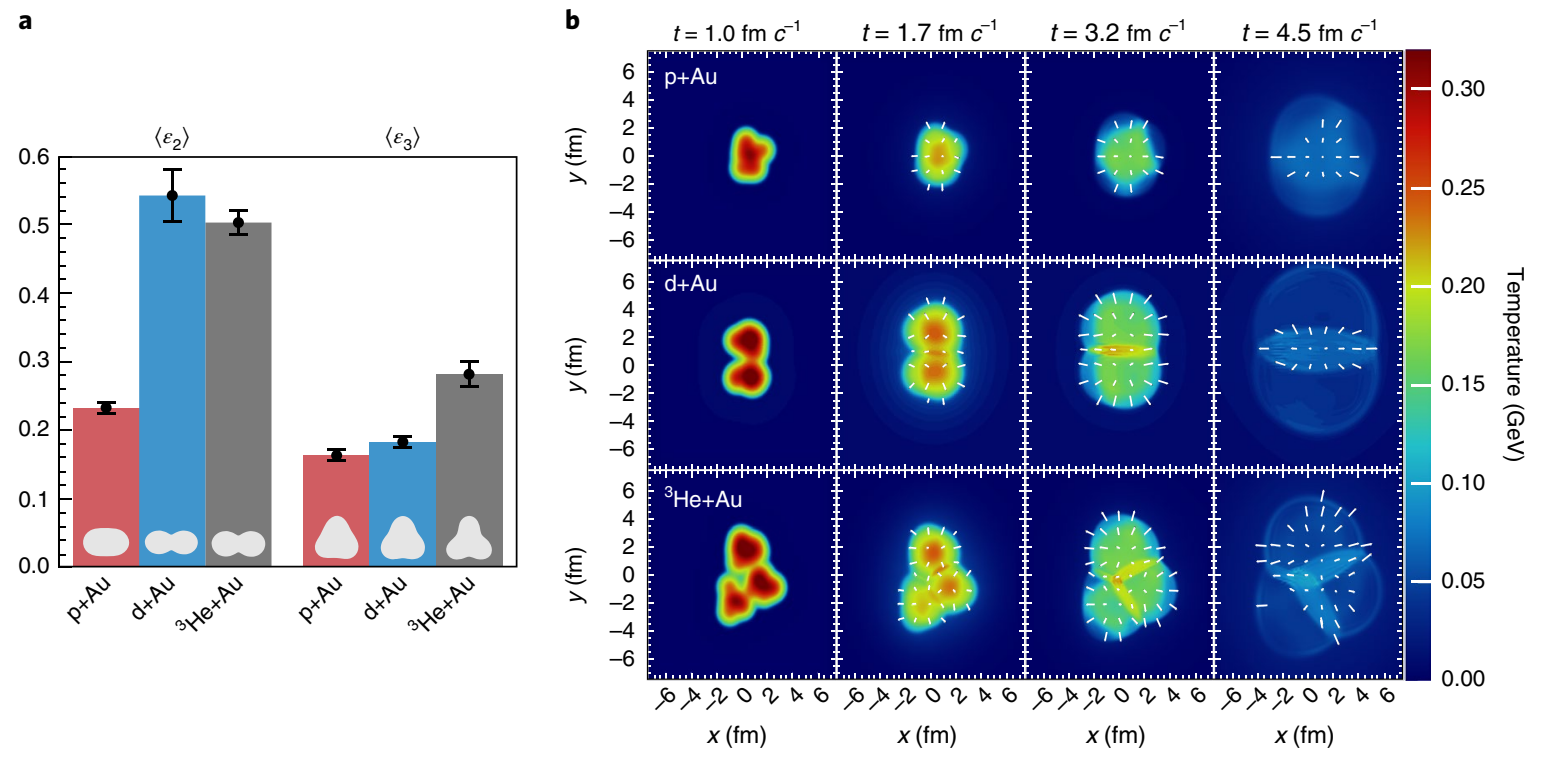

Fig. 1 | Average system eccentricities from a Monte Carlo (MC) Glauber model and hydrodynamic evolution of small systems. a, Average second (third) order spatial eccentricities, $\varepsilon_{2}\left(\varepsilon_{3}\right)$, shown as columns for small impact parameter $\mathrm{p}+\mathrm{Au}(\mathrm{red}), \mathrm{d}+\mathrm{Au}(\mathrm{blue})$ and ${ }^{3} \mathrm{He}+\mathrm{Au}$ (black) collisions as calculated from a MC Glauber model. The second- and third-order spatial eccentricities correspond to ellipticity and triangularity, respectively, as depicted by the shapes inset in the bars. The vertical lines represent one standard deviation systematic uncertainties. $\mathbf{b}$, Hydrodynamic evolution of a typical head-on $\mathrm{p}+$ $\mathrm{Au}$ (top), $\mathrm{d}+\mathrm{Au}$ (middle) and ${ }^{3} \mathrm{He}+\mathrm{Au}$ (bottom) collision at $\sqrt{S_{\mathrm{NN}}}=200 \mathrm{GeV}$ as calculated by SONIC, where the $\mathrm{p} / \mathrm{d} /{ }^{3} \mathrm{He}$ completely overlap with the Au nucleus. From left to right each row gives the temperature distribution of the nuclear matter at four time points following the initial collision at $t=0$. The arrows depict the velocity field, with the length of the longest arrow plotted corresponding to $\beta=0.82$.

clear prediction for the ordering of the experimentally accessible $v_{2}$ and $v_{3}$ signals, following that of the $\varepsilon_{n}$, namely

$$
\begin{aligned}
& v_{2}^{\mathrm{p}+\mathrm{Au}}<v_{2}^{\mathrm{d}+\mathrm{Au}} \approx v_{2}{ }^{3} \mathrm{He}+\mathrm{Au} \\
& v_{3}^{\mathrm{p}+\mathrm{Au}} \approx v_{3}^{\mathrm{d}+\mathrm{Au}}<v_{3}{ }^{3} \mathrm{He}+\mathrm{Au}
\end{aligned}
$$

This ordering assumes that hydrodynamics can efficiently translate the initial geometric $\varepsilon_{n}$ into dynamical $v_{n}$, which in turn requires a small value for the specific shear viscosity.

There exist a class of alternative explanations where $v_{n}$ is not generated via flow, but rather is created at the earliest time in the collision process as described by so-called initial-state momentum correlation models. They produce a mimic flow signal where the initial collision generates colour flux tubes that have a preference to emit particles back-to-back in azimuth ${ }^{19,20}$. These colour flux tubes, also referred to as domains, have a transverse size relative to the collision axis less than the colour-correlation length of order $0.1-0.2 \mathrm{fm}$. In the case where individual domains are resolved, a collision system with a larger overall area but the same characteristic domain size (for example $\mathrm{d}+\mathrm{Au}$ and ${ }^{3} \mathrm{He}+\mathrm{Au}$ compared with $\mathrm{p}+\mathrm{Au}$ and $p+p)$ should have a weaker correlation because the different domains are separated and do not communicate ${ }^{21,22}$. An instructive analogy is a ferromagnet with many domains: if the domains are separated and disconnected, the overall magnetic field is weakened by the cancellation of effects from the random orientation in the different domains. The root-mean-square diameter of the deuteron is $4.2 \mathrm{fm}$, and so in $\mathrm{d}+\mathrm{Au}$ collisions the two hot spots are typically much farther apart than the characteristic domain size. A straightforward prediction is then that the $v_{2}$ and $v_{3}$ coefficients should be ordered

$$
v_{n}^{\mathrm{p}+\mathrm{Au}}>v_{n}^{\mathrm{d}+\mathrm{Au}}>v_{n}{ }^{3} \mathrm{He}+\mathrm{Au}
$$

in contradistinction to the hydrodynamic flow prediction.
An experimental realization of the proposed geometry scan has been under way at the RHIC. Collisions of ${ }^{3} \mathrm{He}+\mathrm{Au}, \mathrm{p}+\mathrm{Au}$ and $\mathrm{d}+\mathrm{Au}$ at $\sqrt{s_{\mathrm{N}}}=200 \mathrm{GeV}$ were recorded in 2014, 2015 and 2016, respectively. The PHENIX experiment observed elliptic anisotropies in the azimuthal distributions of the charged particles produced in all three systems ${ }^{23-25}$, as well as triangular anisotropies in ${ }^{3} \mathrm{He}+\mathrm{Au}$ collisions ${ }^{25}$. This Letter completes this set of elliptic and triangular flow measurements from PHENIX in all three systems and explores the relation between the strength of the measured $v_{n}$ and the initial-state geometry.

The $v_{n}$ measurements reported here are determined using the event plane method ${ }^{26}$ for charged hadrons in the midrapidity region covering $|\eta|<0.35$, where $\eta$ is the particle pseudorapidity

$$
\eta \equiv-\ln \left(\tan \frac{\theta}{2}\right)
$$

and $\theta$ is the polar angle of the particle. The second-order event plane is determined using detectors in the Au-going direction covering $-3.0<\eta<-1.0$ in $\mathrm{p} / \mathrm{d}+\mathrm{Au}$ and $-3.9<\eta<-3.1$ in ${ }^{3} \mathrm{He}+$ $\mathrm{Au}$. The third-order event plane is determined using detectors in the Au-going direction covering $-3.9<\eta<-3.1$ in all cases. The pseudorapidity gap between the particle measurements and the event plane determination excludes autocorrelations and reduces short-range correlations arising from, for example, jets and particle decays-typically referred to as non-flow correlations. Estimates of possible remaining non-flow contributions are included in the systematic uncertainties. Additional uncertainties related to detector alignment, data selection and event plane determination are also included in the systematic uncertainty estimation (see Methods). In these small collision systems the event plane resolution is low, meaning that $v_{n}\{\mathrm{EP}\}=\sqrt{\left\langle v_{n}^{2}\right\rangle}$ (ref. ${ }^{27}$ ) and the results are therefore equivalent to measurements using two-particle correlation methods. 


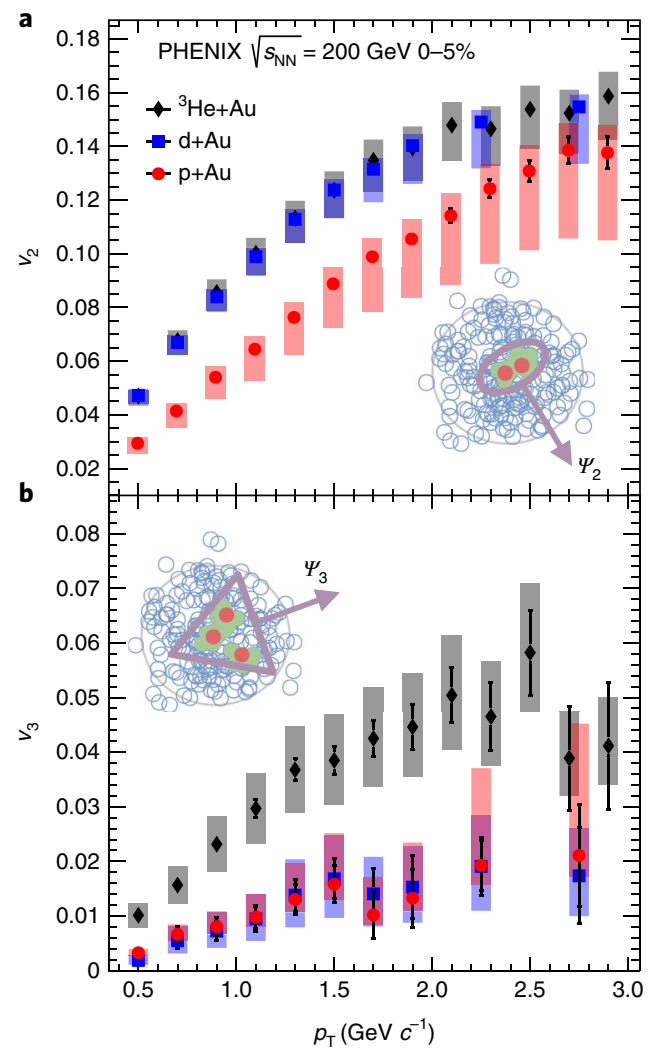

Fig. 2 | Measured $v_{n}\left(p_{T}\right)$ in three collision systems. a, Measurements of $v_{2}\left(p_{T}\right)$ in the $0-5 \%$ most central $p+A u, d+A u$ and ${ }^{3} \mathrm{He}+\mathrm{Au}$ collisions at $\sqrt{S_{N N}}=200 \mathrm{GeV}$. A d+Au event from a MC Glauber model is inset with the elliptic symmetry plane angle, $\psi_{2}$, depicted. b, Measurements of $v_{3}\left(p_{\mathrm{T}}\right)$ in the $0-5 \%$ most central $\mathrm{p}+\mathrm{Au}, \mathrm{d}+\mathrm{Au}$ and ${ }^{3} \mathrm{He}+\mathrm{Au}$ collisions at $\sqrt{S_{\mathrm{NN}}}=200 \mathrm{GeV}$. A ${ }^{3} \mathrm{He}+$ Au event from a MC Glauber model is inset with the triangular symmetry plane angle, $\psi_{3}$, depicted. Each point in a,b represents an average over $p_{\mathrm{T}}$ bins of width $0.2 \mathrm{GeV}^{-1}$ to $0.5 \mathrm{GeV}^{-1}$. The vertical lines (boxes) represent one standard deviation statistical (systematic) uncertainties.

Measurements of $v_{n}$ as a function of $p_{\mathrm{T}}$ are shown for all three systems in Fig. 2. The measurements are performed in the $0-5 \%$ most central events, an experimentally determined criterion that selects the $5 \%$ of events with the largest number of produced particles (hereafter referred to simply as 'multiplicity') in the region $-3.9<\eta<-3.1$. A detailed description of the centrality determination in small systems is given in ref. ${ }^{28}$. The vertical bars on each point represent the statistical uncertainties, while the shaded boxes represent the systematic uncertainties. The flow coefficients follow the prediction of hydrodynamical models shown in equation (3). These relationships suggest that the primary driver of azimuthal momentum anisotropies in particle emission is initial spatial anisotropy.

While Fig. 2 offers qualitative support for the hydrodynamic theory, Fig. 3 directly compares these data to predictions from two hydrodynamical models, SONIC ${ }^{18}$ (used in Fig. 1) and iEBEVISHNU $^{29}$. The core structure of the two models is similar: the initial conditions are evolved using viscous hydrodynamics, the fluid hadronizes, hadronic scattering occurs, and the $v_{n}$ coefficients of the final-state hadron distributions are determined using two-particle correlation methods. However, the detailed implementations are different, including the use of different fluctuations in the initial energy deposited, as well as different hadronic rescattering packages. Both calculations in Fig. 3 use a ratio of the shear viscosity $\eta$ to entropy density $s$ of $\eta / s=0.08 \approx \frac{1}{4 \pi}$, the conjectured lower limit in strongly coupled field theories ${ }^{30}$.

Figure 3 shows that the hydrodynamical models are consistent with the $v_{n}$ data in all three systems. Both models capture the magnitude difference of $v_{3}$ compared to $v_{2}$, the collision system dependence, as well as the general $p_{\mathrm{T}}$ dependence of $v_{3}$. The models tend to diverge at higher $p_{\mathrm{T}}$ in the case of $v_{3}$, which may be more sensitive to the hadronic rescattering. To quantify the agreement, we calculate $p$ values following the procedure of incorporating data systematic uncertainties and their correlations into a modified $\chi^{2}$ analysis laid out in ref. ${ }^{31}$ (see Methods). We find that SONIC and iEBE-VISHNU yield combined $p$ values across the six measurements of 0.90 and 0.14 , respectively. The large difference in $p$ values is driven by the effect of the dominant non-flow uncertainty, which is asymmetric and anti-correlated between $v_{2}$ and $v_{3}$. SONIC tends to underestimate the $v_{2}$ and overestimate the $v_{3}$, particularly in $\mathrm{p}+\mathrm{Au}$ and $\mathrm{d}+\mathrm{Au}$, which is more in line with the uncertainty correlations than iEBEVISHNU, which tends to yield a poorer description of the $p_{\mathrm{T}}$ slope. Overall, the simultaneous description of these two observables in three different systems using a common initial geometry model and the same specific $\eta / s$ strongly supports the hydrodynamic picture.

The hydrodynamic calculations shown in Fig. 3 use initial conditions generated from a nucleon Glauber model. However, initial geometries with quark substructure do not significantly change the $\varepsilon_{2}$ and $\varepsilon_{3}$ values for high multiplicity $\mathrm{p} / \mathrm{d} /{ }^{3} \mathrm{He}+\mathrm{Au}$ collisions ${ }^{32,33}$ and thus the hydrodynamic results should be relatively insensitive to these variations.

While we have focused on hydrodynamical models here, there is an alternative class of models that also translate initial spatial eccentricity to final-state particle azimuthal momentum anisotropy. Instead of hydrodynamic evolution, the translation occurs via parton-parton scattering with a modest interaction cross-section. These parton transport models, for example A Multi-Phase Transport (AMPT) Model $^{34}$, are able to capture the system ordering of $v_{n}$ at low $p_{\mathrm{T}}$ in small systems ${ }^{35}$, but fail to describe the $p_{\mathrm{T}}$ dependence and overall magnitude of the coefficients for all systems resulting in a $p$ value consistent with zero when compared with the data shown here. We have additionally analysed AMPT following the identical PHENIX event plane method and find even worse agreement with the experimental data.

While the initial geometry models for the $\mathrm{d}+\mathrm{Au}$ and ${ }^{3} \mathrm{He}+\mathrm{Au}$ are largely constrained by our detailed understanding of the two-body and three-body nucleon correlations in the deuteron and ${ }^{3} \mathrm{He}$ nuclei, respectively, the distribution of deposited energy around each nucleon-nucleon collision site could result in an ambiguity between the allowed ranges of the $\eta / s$ and the broadening of the initial distribution, as pointed out in ref. ${ }^{13}$. However, a broader distribution of deposited energy results in a significant reduction of the $\varepsilon_{2}$ values and an even greater reduction of $\varepsilon_{3}$, with by far the largest reduction in the $\mathrm{p}+\mathrm{Au}$ system. Here again, the simultaneous constraints of the elliptic and triangular flow ordering eliminates this ambiguity.

Our experimental data also rule out the initial-state correlations scenario where colour domains are individually resolved as the dominant mechanism for creating $v_{2}$ and $v_{3}$ in $\mathrm{p} / \mathrm{d} /{ }^{3} \mathrm{He}+\mathrm{Au}$ collisions. After our results became publicly available, a new calculation was presented in ref. ${ }^{36}$, hereafter referred to as MSTV, where the ordering of the measured $v_{n}$ values matches the experimental data. This calculation posits that gluons from the $\mathrm{Au}$ target do not resolve individual colour domains in the projectile $\mathrm{p} / \mathrm{d} /{ }^{3} \mathrm{He}$ and interact with them coherently, and thus the ordering does not follow equation (4). The MSTV calculations are shown in Fig. 3 and yield a combined $p$ value of effectively zero, in contradistinction to the robust values found for the hydrodynamic models. Another key statement made by MSTV - that in the dilute-dense limit the saturation scale $Q_{s}^{2}$ is proportional to the number of produced charged particles-is questionable ${ }^{37}$, but also leads the MSTV authors to 


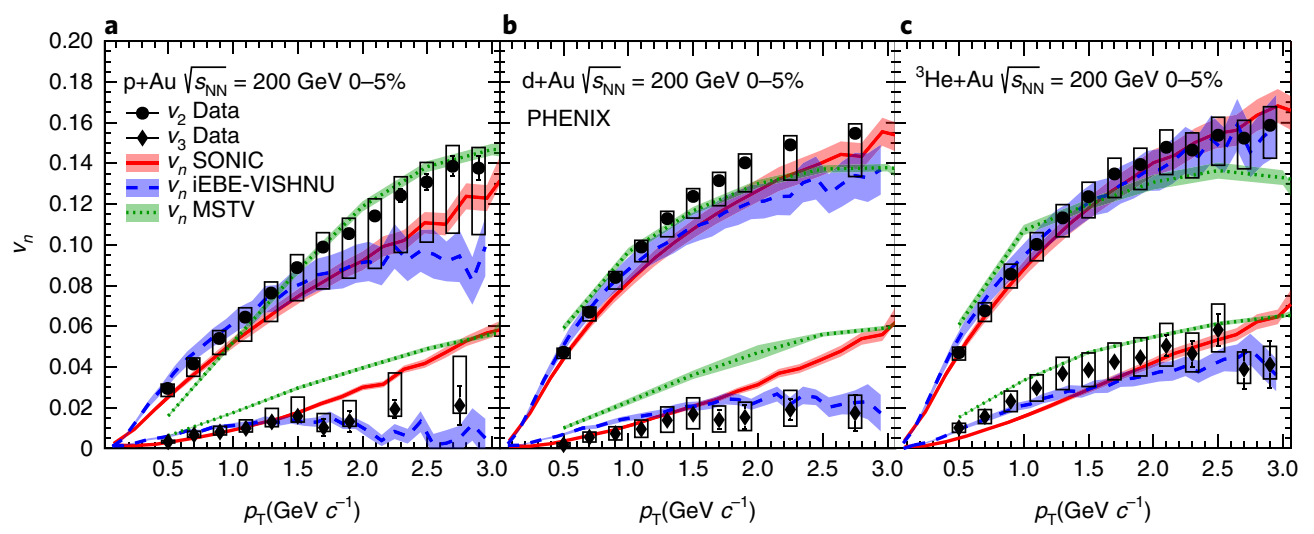

Fig. $3 \mid$ Measured $v_{n}\left(p_{T}\right)$ in three collision systems compared with models. a, Measured $v_{n}\left(p_{T}\right)$ in the 0-5\% most central p+Au collisions compared with models. b. Measured $v_{n}\left(p_{T}\right)$ in the $0-5 \%$ most central d+Au collisions compared with models. c, Measured $v_{n}\left(p_{T}\right)$ in the $0-5 \%$ most central ${ }^{3} \mathrm{He}+\mathrm{Au}$ compared with models. Each point in a-c represents an average over $p_{\mathrm{T}}$ bins of width $0.2 \mathrm{GeV} c^{-1}$ to $0.5 \mathrm{GeV} c^{-1}$; black circles are $v_{2}\left(p_{T}\right)$, black diamonds are $v_{3}\left(p_{T}\right)$. The vertical lines (boxes) represent one standard deviation statistical (systematic) uncertainties. The solid red (dashed blue) curves represent hydrodynamic predictions of $v_{n}\left(p_{T}\right)$ from SONIC (iEBE-VISHNU). The dotted green curves represent initial-state momentum correlation postdictions of $v_{n}\left(p_{T}\right)$ from MSTV.

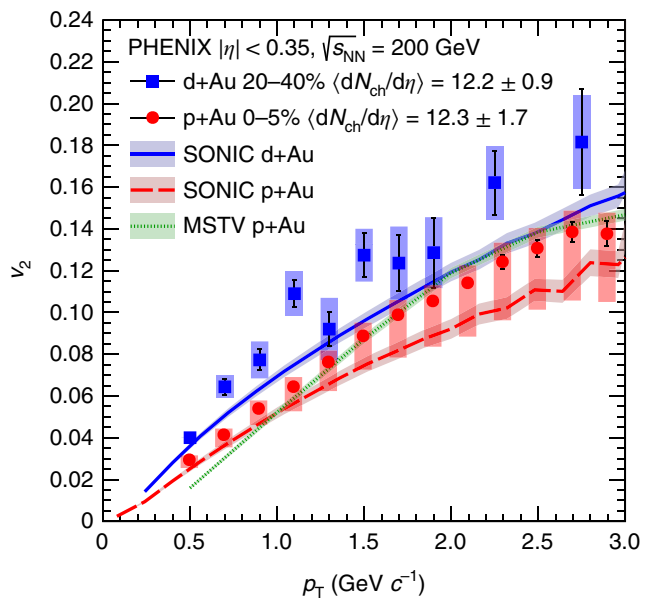

Fig. 4 | Measured $v_{2}\left(p_{T}\right)$ in $p+A u$ and $d+A u$ collisions at the same event multiplicity. Measured $v_{2}\left(p_{T}\right)$ in the $0-5 \%$ most central $p+$ Au collisions and $20-40 \%$ central $d+$ Au collisions compared with SONIC predictions and MSTV postdictions. Each point represents an average over $p_{\mathrm{T}}$ bins of width $0.2 \mathrm{GeV}^{-1}$ to $0.5 \mathrm{GeV}^{-1}$. The vertical lines (boxes) represent one standard deviation statistical (systematic) uncertainties. The quoted $\mathrm{d} N_{\mathrm{ch}} / \mathrm{d} \eta$ values are taken from ref. ${ }^{38}$. Blue and red curves correspond to SONIC predictions for $d+A u$ and $p+A u$, respectively. The green curve corresponds to MSTV calculations for $0-5 \%$ central $p+A u$ collisions, which the authors state yield an identical $v_{2}\left(p_{T}\right)$ for $d+$ Au collisions at the same multiplicity.

make a clear prediction that the $v_{2}$ will be identical between systems when selecting on the same event multiplicity. Shown in Fig. 4 are the previously published $\mathrm{d}+\mathrm{Au}(20-40 \%)$ and $\mathrm{p}+\mathrm{Au}(0-5 \%)$ $v_{2}$ where the measured mean charged particle multiplicities $\left(\mathrm{d} N_{\mathrm{ch}} / \mathrm{d} \eta\right)$ match $^{38}$. The results do not support the MSTV prediction of an identical $v_{2}$ for these two systems at the same multiplicity, while the differences in $v_{2}$ between the systems follow the expectations from hydrodynamic calculations matched to the same $\mathrm{d} N_{\mathrm{ch}} / \mathrm{d} \eta$.

The simultaneous constraints of $v_{2}$ and $v_{3}$ in $\mathrm{p} / \mathrm{d} /{ }^{3} \mathrm{He}+\mathrm{Au}$ collisions definitively demonstrate that the $v_{n}$ coefficients are correlated with the initial geometry, removing ambiguities related to event multiplicity and initial event geometry. Further, hydrodynamical models that include QGP formation provide a simultaneous and quantitative description of the data in all three systems. Similar small-system geometry tests performed at the LHC, which provide an order of magnitude increase in collision energy, would be insightful particularly by enabling higher statistics, multi-particle correlation observables.

\section{Online content}

Any methods, additional references, Nature Research reporting summaries, source data, statements of data availability and associated accession codes are available at https://doi.org/10.1038/ s41567-018-0360-0.

Received: 8 May 2018; Accepted: 25 October 2018; Published online: 10 December 2018

\section{References}

1. Arsene, I. et al. Quark gluon plasma and color glass condensate at RHIC? The perspective from the BRAHMS experiment. Nucl. Phys. A 757, 1-27 (2005).

2. Back, B. B. et al. The PHOBOS perspective on discoveries at RHIC. Nucl. Phys. A 757, 28-101 (2005).

3. Adams, J. et al. Experimental and theoretical challenges in the search for the quark gluon plasma: the STAR Collaboration's critical assessment of the evidence from RHIC collisions. Nucl. Phys. A 757, 102-183 (2005).

4. Adcox, K. et al. Formation of dense partonic matter in relativistic nucleusnucleus collisions at RHIC: experimental evaluation by the PHENIX collaboration. Nucl. Phys. A 757, 184-283 (2005).

5. Heinz, U. \& Snellings, R. Collective flow and viscosity in relativistic heavy-ion collisions. Annu. Rev. Nucl. Part. Sci. 63, 123-151 (2013).

6. Khachatryan, V. et al. Observation of long-range near-side angular correlations in proton-proton collisions at the LHC. J. High Energy Phys. 09, 091 (2010).

7. Chatrchyan, S. et al. Observation of long-range near-side angular correlations in proton-lead collisions at the LHC. Phys. Lett. B 718, 795-814 (2013).

8. Abelev, B. et al. Long-range angular correlations on the near and away side in $p$ - $\mathrm{Pb}$ collisions at $=5.02 \mathrm{TeV}$. Phys. Lett. B 719, 29-41 (2013).

9. Aad, G. et al. Observation of associated near-side and away-side long-range correlations in $=5.02 \mathrm{TeV}$ proton-lead collisions with the ATLAS detector. Phys. Rev. Lett. 110, 182302 (2013).

10. Adare, A. et al. Quadrupole anisotropy in dihadron azimuthal correlations in central $d+$ Au collisions at $=200 \mathrm{GeV}$. Phys. Rev. Lett. 111, 212301 (2013).

11. Adare, A. et al. Measurement of long-range angular correlation and quadrupole anisotropy of pions and (anti)protons in central $d+$ Au collisions at $=200 \mathrm{GeV}$. Phys. Rev. Lett. 114, 192301 (2015). 
12. Dusling, K., Li, W. \& Schenke, B. Novel collective phenomena in high-energy proton-proton and proton-nucleus collisions. Int. J. Mod. Phys. E 25, 1630002 (2016).

13. Nagle, J. L. \& Zajc, W. A. Small system collectivity in relativistic hadron and nuclear collisions. Preprint at https://arXiv.org/abs/1801.03477v2 (2018).

14. Nagle, J. L. et al. Exploiting intrinsic triangular geometry in relativistic ${ }^{3} \mathrm{He}+\mathrm{Au}$ collisions to disentangle medium properties. Phys. Rev. Lett. 113, 112301 (2014).

15. Miller, M. L., Reygers, K., Sanders, S. J. \& Steinberg, P. Glauber modeling in high energy nuclear collisions. Annu. Rev. Nucl. Part. Sci. 57, 205-243 (2007).

16. Alver, B. \& Roland, G. Collision geometry fluctuations and triangular flow in heavy-ion collisions. Phys. Rev. C 81, 054905 (2010); erratum 82, 039903 (2010).

17. Gale, C., Jeon, S. \& Schenke, B. Hydrodynamic modeling of heavy-ion collisions. Int. J. Mod. Phys. A 28, 1340011 (2013).

18. Habich, M., Nagle, J. L. \& Romatschke, P. Particle spectra and HBT radii for simulated central nuclear collisions of $\mathrm{C}+\mathrm{C}, \mathrm{Al}+\mathrm{Al}, \mathrm{Cu}+\mathrm{Cu}, \mathrm{Au}+\mathrm{Au}$, and $\mathrm{Pb}+\mathrm{Pb}$ from $=62.4-2760 \mathrm{GeV}$. Eur. Phys. J. C 75, 15 (2015).

19. Dusling, K. \& Venugopalan, R. Azimuthal collimation of long range rapidity correlations by strong color fields in high multiplicity hadron-hadron collisions. Phys. Rev. Lett. 108, 262001 (2012).

20. Dumitru, A. et al. The ridge in proton-proton collisions at the LHC. Phys. Lett. B 697, 21-25 (2011).

21. Dumitru, A. \& Giannini, A. V. Initial state angular asymmetries in high energy $p+$ A collisions: spontaneous breaking of rotational symmetry by a color electric field and C-odd fluctuations. Nucl. Phys. A 933, 212-228 (2015).

22. Lappi, T., Schenke, B., Schlichting, S. \& Venugopalan, R. Tracing the origin of azimuthal gluon correlations in the color glass condensate. J. High Energy Phys. 01, 061 (2016).

23. Aidala, C. et al. Measurement of long-range angular correlations and azimuthal anisotropies in high-multiplicity $p+\mathrm{Au}$ collisions at $=200 \mathrm{GeV}$. Phys. Rev. C 95, 034910 (2017).

24. Aidala, C. et al. Measurements of azimuthal anisotropy and charged-particle multiplicity in $d+$ Au collisions at $=200,62.4,39$, and $19.6 \mathrm{GeV}$. Phys. Rev. C 96, 064905 (2017).

25. Adare, A. et al. Measurements of elliptic and triangular flow in high-multiplicity ${ }^{3} \mathrm{He}+$ Au collisions at $=200 \mathrm{GeV}$. Phys. Rev. Lett. 115, 142301 (2015).

26. Voloshin, S. \& Zhang, Y. Flow study in relativistic nuclear collisions by Fourier expansion of azimuthal particle distributions. Z. Phys. C 70, 665-672 (1996).

27. Ollitrault, J.-Y., Poskanzer, A. M. \& Voloshin, S. A. Effect of nonflow and flow fluctuations on elliptic flow methods. Nucl. Phys. A 830, 279c-282c (2009).

28. Adare, A. et al. Centrality categorization for $R_{p(d)+A}$ in high-energy collisions. Phys. Rev. C 90, 034902 (2014).

29. Shen, C., Paquet, J.-F., Denicol, G. S., Jeon, S. \& Gale, C. Collectivity and electromagnetic radiation in small systems. Phys. Rev. C 95, 014906 (2017).

30. Kovtun, P., Son, D. T. \& Starinets, A. O. Viscosity in strongly interacting quantum field theories from black hole physics. Phys. Rev. Lett. 94, 111601 (2005).

31. Adare, A. et al. Quantitative constraints on the opacity of hot partonic matter from semi-inclusive single high transverse momentum pion suppression in $\mathrm{Au}+\mathrm{Au}$ collisions at $=200 \mathrm{GeV}$. Phys. Rev. C 77, 064907 (2008).

32. Welsh, K., Singer, J. \& Heinz, U. W. Initial state fluctuations in collisions between light and heavy ions. Phys. Rev. C 94, 024919 (2016).

33. Weller, R. D. \& Romatschke, P. One fluid to rule them all: viscous hydrodynamic description of event-by-event central $p+p, p+\mathrm{Pb}$ and $\mathrm{Pb}+\mathrm{Pb}$ collisions at $=5.02 \mathrm{TeV}$. Phys. Lett. B 774, 351-356 (2017).
34. Lin, Z.-W., Ko, C. M., Li, B.-A., Zhang, B. \& Pal, S. A multi-phase transport model for relativistic heavy ion collisions. Phys. Rev. C 72, 064901 (2005).

35. Orjuela Koop, J. D., Adare, A., McGlinchey, D. \& Nagle, J. L. Azimuthal anisotropy relative to the participant plane from a multiphase transport model in central $p+\mathrm{Au}, d+\mathrm{Au}$, and ${ }^{3} \mathrm{He}+\mathrm{Au}$ collisions at $=200 \mathrm{GeV}$. Phys. Rev. C 92, 054903 (2015).

36. Mace, M., Skokov, V. V., Tribedy, P. \& Venugopalan, R. Hierarchy of azimuthal anisotropy harmonics in collisions of small systems from the color glass condensate. Phys. Rev. Lett. 121, 052301 (2018).

37. Nagle, J. L. \& Zajc, W. A. Assessing saturation physics explanations of collectivity in small collision systems with the IP-Jazma model. Preprint at https://arXiv.org/abs/1808.01276v1 (2018).

38. Adare, A. et al. Pseudorapidity dependence of particle production and elliptic flow in asymmetric nuclear collisions of $p+\mathrm{Al}, p+\mathrm{Au}, d+\mathrm{Au}$, and ${ }^{3} \mathrm{He}+\mathrm{Au}$ at $\sqrt{s_{N N}}=200 \mathrm{GeV}$. Preprint at https://arXiv.org/abs/1807.11928v1 (2018).

\section{Acknowledgements}

We thank the staff of the Collider-Accelerator and Physics Departments at Brookhaven National Laboratory and the staff of the other PHENIX participating institutions for their vital contributions. We acknowledge support from the Office of Nuclear Physics in the Office of Science of the Department of Energy, the National Science Foundation, Abilene Christian University Research Council, Research Foundation of SUNY, and Dean of the College of Arts and Sciences, Vanderbilt University (USA), Ministry of Education, Culture, Sports, Science, and Technology and the Japan Society for the Promotion of Science (Japan), Conselho Nacional de Desenvolvimento Cientfico e Tecnológico and Fundação de Amparo à Pesquisa do Estado de São Paulo (Brazil), Natural Science Foundation of China (People's Republic of China), Croatian Science Foundation and Ministry of Science and Education (Croatia), Ministry of Education, Youth and Sports (Czech Republic), Centre National de la Recherche Scientifique, Commissariat à l'Énergie Atomique, and Institut National de Physique Nucléaire et de Physique des Particules (France), Bundesministerium für Bildung und Forschung, Deutscher Akademischer Austausch Dienst, and Alexander von Humboldt Stiftung (Germany), NKFIH, EFOP, the New National Excellence Program (ÚNKP) and the J. Bolyai Research Scholarships (Hungary), Department of Atomic Energy and Department of Science and Technology (India), Israel Science Foundation (Israel), Basic Science Research Program through NRF of the Ministry of Education (Korea), Physics Department, Lahore University of Management Sciences (Pakistan), Ministry of Education and Science, Russian Academy of Sciences, Federal Agency of Atomic Energy (Russia), VR and Wallenberg Foundation (Sweden), the US Civilian Research and Development Foundation for the Independent States of the Former Soviet Union, the Hungarian American Enterprise Scholarship Fund, and the US-Israel Binational Science Foundation.

\section{Author contributions}

All PHENIX collaboration members contributed to the publication of these results in a variety of roles including detector construction, data collection, data processing, and analysis. A subset of collaboration members prepared this manuscript, and all authors had the opportunity to review the final version.

\section{Competing interests}

The authors declare no competing interests. 


\section{PHENIX Collaboration}

C. Aidala', Y. Akiba ${ }^{2,3,67}$, M. Alfred ${ }^{4}$, V. Andrieux', K. Aoki ${ }^{5}$, N. Apadula6, H. Asano ${ }^{2,7}$, C. Ayuso', B. Azmoun ${ }^{8}$, V.Babintsev9 , A. Bagoly ${ }^{10}$, N. S. Bandara"1, K. N. Barish"12, S. Bathe ${ }^{3,13}$, A. Bazilevsky ${ }^{8}$, M. Beaumier ${ }^{12}$, R. Belmont ${ }^{14}$, A. Berdnikov ${ }^{15}$, Y. Berdnikov ${ }^{15}$, D. S. Blau ${ }^{16,17}$, M. Boer ${ }^{18}$, J. S. Bok ${ }^{19}$, M. L. Brooks ${ }^{18}$, J. Bryslawskyj ${ }^{12,13}$, V. Bumazhnov ${ }^{9}$, C. Butler ${ }^{20}$, S. Campbell ${ }^{21,22}$, V. Canoa Roman ${ }^{23}$, R. Cervantes ${ }^{23}$, C. Y. Chi ${ }^{21}$, M. Chiu ${ }^{8}$, I. J. Choi ${ }^{24}$, J. B. Choi ${ }^{25,68}$, Z. Citron ${ }^{26}$, M. Connors ${ }^{3,20}$, N. Cronin ${ }^{23}$, M. Csanád ${ }^{10}$, T. Csörgő ${ }^{27,28}$, T.W. Danley ${ }^{29}$, M. S. Daugherity ${ }^{30}$, G. David ${ }^{8,23}$, K. DeBlasio ${ }^{31}$, K. Dehmelt ${ }^{23}$, A. Denisov ${ }^{9}$, A. Deshpande, 3,23, E. J. Desmond ${ }^{8}$, A. Dion ${ }^{23}$, D. Dixit ${ }^{23}$, L. D. Liu ${ }^{32}$, J. H. Do ${ }^{33}$, A. Drees ${ }^{23}$, K. A.Drees ${ }^{34}$, M. Dumancic ${ }^{26}$, J. M. Durham ${ }^{18}$, A. Durum ${ }^{9}$, T. Elder ${ }^{20}$, A. Enokizono ${ }^{2,35}$, H. En'yo², S. Esumi ${ }^{36}$, B. Fadem ${ }^{37}$, W. Fan ${ }^{23}$, N. Feege ${ }^{23}$, D. E. Fields ${ }^{31}$, M. Finger ${ }^{38}$, M. Finger Jr ${ }^{38}$, S. L. Fokin ${ }^{16}$, J. E. Frantz ${ }^{29}$, A.Franz ${ }^{8}$, A. D. Frawley ${ }^{39}$, Y. Fukuda ${ }^{36}$, C. Gal ${ }^{23}$, P.Gallus ${ }^{40}$, P. Garg ${ }^{23,41}$, H. Ge ${ }^{23}$, F. Giordano ${ }^{24}$, Y. Goto ${ }^{2,3}$, N. Grau42, S. V. Greene ${ }^{43}$, M. Grosse Perdekamp ${ }^{24}$, T. Gunji ${ }^{44}$, H. Guragain ${ }^{20}$, T. Hachiya ${ }^{2,3}$, J. S. Haggerty ${ }^{8}$, K. I. Hahn ${ }^{45}$, H. Hamagaki ${ }^{44}$, H. F. Hamilton ${ }^{30}$, S. Y. Han ${ }^{45}$, J. Hanks ${ }^{23}$, S. Hasegawa ${ }^{46}$, T. O.S. Haseler ${ }^{20}$, X. He ${ }^{20}$, T. K. Hemmick ${ }^{23}$, J. C. Hill ${ }^{6}$, K. Hill ${ }^{14}$, A. Hodges ${ }^{20}$, R. S. Hollis ${ }^{12}$, K. Homma ${ }^{47}$, B. Hong ${ }^{48}$, T. Hoshino ${ }^{47}$, N. Hotvedt ${ }^{6}$, J. Huang ${ }^{8}$, S. Huang ${ }^{43}$, K. Imai ${ }^{46}$, J. Imrek ${ }^{49}$, M. Inaba ${ }^{36}$, A. Iordanova ${ }^{12}$, D. Isenhower ${ }^{30}$, Y. Ito ${ }^{50}$, D. Ivanishchev ${ }^{51}$, B. V. Jacak ${ }^{23}$, M. Jezghani ${ }^{20}$, Z. Ji ${ }^{23}$, X. Jiang ${ }^{18}$, B. M. Johnson ${ }^{8,20}$, V. Jorjadze ${ }^{23}$, D. Jouan ${ }^{52}$, D. S. Jumper ${ }^{24}$, J. H. Kang 33, D. Kapukchyan'12, S. Karthas ${ }^{23}$, D. Kawall ${ }^{11}$, A. V. Kazantsev ${ }^{16}$, V. Khachatryan ${ }^{23}$, A. Khanzadeev ${ }^{51}$, C. Kim ${ }^{12,48}$, D. J. Kim ${ }^{53}$, E.-J. Kim ${ }^{25}$, M. Kim ${ }^{54}$, M. H. Kim ${ }^{48}$, D. Kincses ${ }^{10}$, E. Kistenev8, J. Klatsky ${ }^{39}$, P. Kline ${ }^{23}$, T. Koblesky ${ }^{14}$, D. Kotov ${ }^{15,51}$, S. Kudo ${ }^{36}$, B. Kurgyis ${ }^{10}$, K. Kurita ${ }^{35}$, Y. Kwon ${ }^{33}$, J. G. Lajoie ${ }^{6}$, E. O. Lallow ${ }^{37}$, A. Lebedev 6 , S. Lee ${ }^{33}$, S. H. Lee ${ }^{6,23}$, M. J. Leitch ${ }^{18}$, Y. H. Leung ${ }^{23}$, N. A. Lewis' ${ }^{1}$, X. Li ${ }^{18}$, S. H. Lim¹8,33, M. X. Liu' ${ }^{18}$, V.-R. Loggins ${ }^{24}$, S. Lökös ${ }^{10,27}$, K. Lovasz ${ }^{49}$, D. Lynch ${ }^{8}$, T. Majoros ${ }^{49}$, Y. I. Makdisi ${ }^{34}$, M. Makek ${ }^{55}$, M. Malaev ${ }^{51}$, V. I. Manko ${ }^{16}$, E. Mannel ${ }^{8}$, H. Masuda ${ }^{35}$, M. McCumber ${ }^{18}$, P. L. McGaughey ${ }^{18}$, D. McGlinchey ${ }^{14,18}$, C. McKinney ${ }^{24}$, M. Mendoza ${ }^{12}$, A. C. Mignerey ${ }^{56}$, D. E. Mihalik ${ }^{23}$, A. Milov' ${ }^{26}$, D. K. Mishra57, J. T. Mitchell ${ }^{8}$, G. Mitsuka ${ }^{3}$, S. Miyasaka2,58, S. Mizuno ${ }^{2,36}$, P. Montuenga ${ }^{24}$, T. Moon ${ }^{33}$, D. P. Morrison ${ }^{8}$, S. I. Morrow ${ }^{43}$, T. Murakami ${ }^{2,7}$, J. Murata ${ }^{2,35}$, K. Nagai ${ }^{58}$, K. Nagashima ${ }^{47}$, T. Nagashima35 , J. L. Nagle ${ }^{14 \star}$, M. I. Nagy ${ }^{10}$, I. Nakagawa ${ }^{2,3}$, H. Nakagomi ${ }^{2,36}$, K. Nakano ${ }^{2,58}$, C. Nattrass ${ }^{59}$, T. Niida ${ }^{36}$, R. Nouicer ${ }^{3,8}$, T. Novák ${ }^{27,28}$, N. Novitzky ${ }^{23}$, R. Novotny ${ }^{40}$, A.S. Nyanin ${ }^{16}$, E. O'Brien ${ }^{8}$, C. A. Ogilvie ${ }^{6}$, J. D. Orjuela Koop ${ }^{14}$, J. D. Osborn', A. Oskarsson ${ }^{60}$, G. J. Ottino ${ }^{31}$, K. Ozawa ${ }^{5,36}$, V.Pantuev61, V. Papavassiliou ${ }^{19}$, J. S. Park ${ }^{54}$, S. Park ${ }^{2,23,54}$, S. F. Pate ${ }^{19}$, M. Patel ${ }^{6}$, W. Peng ${ }^{43}$, D. V. Perepelitsa ${ }^{8,14}$, G. D. N. Perera ${ }^{19}$, D. Yu. Peressounko ${ }^{16}$, C. E. PerezLara ${ }^{23}$, J. Perry ${ }^{6}$, R. Petti ${ }^{8}$, M.Phipps ${ }^{8,24}$, C. Pinkenburg ${ }^{8}$, R. P. Pisani ${ }^{8}$, A. Pun ${ }^{29}$, M. L. Purschke ${ }^{8}$, P. V. Radzevich ${ }^{15}$, K. F. Read ${ }^{59,62}$, D. Reynolds ${ }^{63}$, V. Riabov ${ }^{17,51}$, Y. Riabov ${ }^{15,51}$, D. Richford ${ }^{13}$, T. Rinn ${ }^{6}$,

S. D. Rolnick ${ }^{12}$, M. Rosati ${ }^{6}$, Z. Rowan ${ }^{13}$, J. Runchey ${ }^{6}$, A.S.Safonov ${ }^{15}$, T. Sakaguchi ${ }^{8}$, H. Sako ${ }^{46}$, V.Samsonov ${ }^{17,51}$, M.Sarsour ${ }^{20}$, K. Sato ${ }^{36}$, S. Sato ${ }^{46}$, B. Schaefer ${ }^{43}$, B. K. Schmoll ${ }^{59}$, K. Sedgwick ${ }^{12}$, R.Seidl2,3, A.Sen ${ }^{6,59}$, R.Seto ${ }^{12}$, A.Sexton ${ }^{56}$, D.Sharma ${ }^{23}$, I.Shein ${ }^{9}$, T.-A.Shibata ${ }^{2,58}$, K. Shigaki ${ }^{47}$, M. Shimomura ${ }^{6,50}$, T. Shioya ${ }^{36}$, P.Shukla ${ }^{57}$, A. Sickles ${ }^{24}$, C. L. Silva ${ }^{18}$, D. Silvermyr ${ }^{60}$, B. K. Singh ${ }^{41}$, C.P.Singh ${ }^{41}$, V.Singh ${ }^{41}$, M. J.Skoby ${ }^{1}$, M.Slunečka ${ }^{38}$, K. L.Smith ${ }^{39}$, M.Snowball ${ }^{18}$, R. A. Soltz ${ }^{64}$, W. E. Sondheim ${ }^{18}$, S.P. Sorensen ${ }^{59}$, I. V. Sourikova ${ }^{8}$, P. W.Stankus ${ }^{62}$, S. P. Stoll ${ }^{8}$, T. Sugitate ${ }^{47}$, A.Sukhanov ${ }^{8}$, T.Sumita2 ${ }^{2}$ J. Sun ${ }^{23}$, Z. Sun ${ }^{49}$, S.Syed ${ }^{20}$, J.Sziklai ${ }^{28}$, A. Takeda ${ }^{50}$, K. Tanida ${ }^{3,46,54}$, M. J. Tannenbaum ${ }^{8}$, S. Tarafdar ${ }^{26,43}$, A. Taranenko ${ }^{17}$, G. Tarnai ${ }^{49}$, R. Tieulent ${ }^{20,65}$, A. Timilsina6 ${ }^{6}$, T. Todoroki ${ }^{36}$, M. Tomášek ${ }^{40}$, C. L. Towell ${ }^{30}$, R. S. Towell ${ }^{30}$, I. Tserruya ${ }^{26}$, Y. Ueda ${ }^{47}$, B. Ujvari ${ }^{49}$, H.W.van Hecke ${ }^{18}$, S. Vazquez-Carson ${ }^{14}$, J.Velkovska ${ }^{43}$, M. Virius ${ }^{40}$, V. Vrba ${ }^{40,66}$, N. Vukman ${ }^{55}$, X. R. Wang ${ }^{3,19}$, 


\section{Z. Wang ${ }^{13}$, Y. Watanabe ${ }^{2,3}$, Y.S. Watanabe ${ }^{44}$, C.P. Wong ${ }^{20}$, C. L. Woody ${ }^{8}$, C.Xu' ${ }^{19}$, Q.Xu' ${ }^{43}$, L.Xue ${ }^{20}$, S. Yalcin ${ }^{23}$, Y. L. Yamaguchi ${ }^{3,23}$, H. Yamamoto ${ }^{36}$, A. Yanovich ${ }^{9}$, P. Yin ${ }^{14}$, J.H. Yoo ${ }^{48}$, I. Yoon ${ }^{54}$, H. Yu ${ }^{19,32}$, I. E. Yushmanov ${ }^{16}$, W. A.Zajc ${ }^{21}$, A. Zelenski ${ }^{34}$, S. Zharko ${ }^{15}$ and L.Zou' ${ }^{12}$}

'Department of Physics, University of Michigan, Ann Arbor, MI, USA. ${ }^{2}$ IIKEN Nishina Center for Accelerator-Based Science, Wako, Saitama, Japan. ${ }^{3}$ RIKEN BNL Research Center, Brookhaven National Laboratory, Upton, NY, USA. ${ }^{4}$ Department of Physics and Astronomy, Howard University, Washington, DC, USA. ${ }^{5}$ KEK, High Energy Accelerator Research Organization, Tsukuba, Ibaraki, Japan. ${ }^{6}$ lowa State University, Ames, IA, USA. ${ }^{7}$ Kyoto University, Kyoto, Japan. ${ }^{8}$ Physics Department, Brookhaven National Laboratory, Upton, NY, USA. ${ }^{9}$ IHEP Protvino, State Research Center of Russian Federation, Institute for High Energy Physics, Protvino, Russia. ${ }^{10} E L T E$, Eötvös Loránd University, Budapest, Hungary. "Department of Physics, University of Massachusetts, Amherst, MA, USA. ${ }^{12}$ University of California-Riverside, Riverside, CA, USA. ${ }^{13}$ Baruch College, City University of New York, New York, NY, USA. ${ }^{14}$ University of Colorado, Boulder, CO, USA. ${ }^{15}$ Saint Petersburg State Polytechnic University, St. Petersburg, Russia. ${ }^{16}$ National Research Center 'Kurchatov Institute', Moscow, Russia. ${ }^{17}$ National Research Nuclear University, MEPhl, Moscow Engineering Physics Institute, Moscow, Russia. ${ }^{18}$ Los Alamos National Laboratory, Los Alamos, NM, USA. ${ }^{19}$ New Mexico State University, Las Cruces, NM, USA. ${ }^{20}$ Georgia State University, Atlanta, GA, USA. ${ }^{21}$ Columbia University, New York, NY, USA. ${ }^{22}$ Nevis Laboratories, Irvington, NY, USA. ${ }^{23}$ Department of Physics and Astronomy, Stony Brook University, SUNY, Stony Brook, NY, USA. ${ }^{24}$ University of Illinois at Urbana-Champaign, Urbana, IL, USA. ${ }^{25}$ Chonbuk National University, Jeonju, Korea. ${ }^{26}$ Weizmann Institute, Rehovot, Israel. ${ }^{27}$ Eszterházy Károly University, Károly Róbert Campus, Gyöngyös, Hungary. ${ }^{28}$ Institute for Particle and Nuclear Physics, Wigner Research Centre for Physics, Hungarian Academy of Sciences (Wigner RCP, RMKI), Budapest, Hungary. ${ }^{29}$ Department of Physics and Astronomy, Ohio University, Athens, $\mathrm{OH}$, USA. ${ }^{30}$ Abilene Christian University, Abilene, TX, USA. ${ }^{31}$ University of New Mexico, Albuquerque, NM, USA. ${ }^{32}$ Peking University, Beijing, People's Republic of China. ${ }^{33}$ Yonsei University, IPAP, Seoul, Korea. ${ }^{34}$ Collider-Accelerator Department, Brookhaven National Laboratory, Upton, NY, USA. ${ }^{35}$ Physics Department, Rikkyo University, Toshima, Tokyo, Japan. ${ }^{36}$ Tomonaga Center for the History of the Universe, University of Tsukuba, Tsukuba, Ibaraki, Japan. ${ }^{37}$ Muhlenberg College, Allentown, PA, USA. ${ }^{38} \mathrm{Charles}$ University, Prague, Czech Republic. ${ }^{39}$ Florida State University, Tallahassee, FL, USA. ${ }^{40} \mathrm{Czech}$ Technical University, Prague 6, Czech Republic. ${ }^{4}$ Department of Physics, Banaras Hindu University, Varanasi, India. ${ }^{42}$ Department of Physics, Augustana University, Sioux Falls, SD, USA. ${ }^{43}$ Vanderbilt University, Nashville, TN, USA. ${ }^{44}$ Center for Nuclear Study, Graduate School of Science, University of Tokyo, Bunkyo, Tokyo, Japan. ${ }^{45}$ Ewha Womans University, Seoul, Korea. ${ }^{46}$ Advanced Science Research Center, Japan Atomic Energy Agency, Naka-gun, Ibaraki-ken, Japan. ${ }^{47}$ Hiroshima University, Kagamiyama, Higashi-Hiroshima, Japan. ${ }^{48}$ Korea University, Seoul, Korea. ${ }^{49}$ Debrecen University, Debrecen, Hungary. ${ }^{50}$ Nara Women's University, Nara, Japan. ${ }^{51}$ PNPI, Petersburg Nuclear Physics Institute, Gatchina, Leningrad region, Russia. ${ }^{52}$ IPN-Orsay, Univ. Paris-Sud, CNRS/ IN2P3, Université Paris-Saclay, Orsay, France. ${ }^{53} \mathrm{Helsinki}$ Institute of Physics and University of Jyväskylä, Jyväskylä, Finland. ${ }^{54}$ Department of Physics and Astronomy, Seoul National University, Seoul, Korea. ${ }^{55}$ Department of Physics, Faculty of Science, University of Zagreb, Zagreb, Croatia. ${ }^{56}$ University of Maryland, College Park, MD, USA. ${ }^{57}$ Bhabha Atomic Research Centre, Bombay, India. ${ }^{58}$ Department of Physics, Tokyo Institute of Technology, Oh-okayama, Meguro, Tokyo, Japan. ${ }^{5}$ University of Tennessee, Knoxville, TN, USA. ${ }^{60}$ Department of Physics, Lund University, Lund, Sweden. ${ }^{61}$ Institute for Nuclear Research of the Russian Academy of Sciences, Moscow, Russia. ${ }^{62}$ Oak Ridge National Laboratory, Oak Ridge, TN, USA. ${ }^{63}$ Chemistry Department, Stony Brook University, SUNY, Stony Brook, New York, NY, USA. ${ }^{64}$ Lawrence Livermore National Laboratory, Livermore, CA, USA. ${ }^{65}$ IPNL, CNRS/IN2P3, Université Lyon, Villeurbanne, France. ${ }^{66}$ Institute of Physics, Academy of Sciences of the Czech Republic, Prague, Czech Republic. ${ }^{67} \mathrm{PHENIX} \mathrm{Spokesperson:}$ akiba@rcf.rhic.bnl.gov: Y. Akiba. ${ }^{68}$ Deceased: J. B. Choi. 


\section{Methods}

Here we provide details of the $v_{3}$ measurements in $\mathrm{p}+\mathrm{Au}$ and $\mathrm{d}+\mathrm{Au}$ collisions as well as details on quantifying comparisons of theory to data. For details on the remaining measurements see refs ${ }^{23-25}$.

Experimental set-up. These measurements utilize the PHENIX detector at the RHIC. Particle tracking is performed by two arms at midrapidity, each covering $|\eta|<0.35$ and $\frac{\pi}{-}$ in azimuth using drift chambers (DC) and pad chambers $(\mathrm{PC})^{39}$. Beam-beam counters (BBC) located at forward and backward rapidities $(3.1<|\eta|<3.9)$, each consisting of an array of 64 quartz Cherenkov radiators readout by photomultiplier tubes ${ }^{28}$, provide event triggering, collision vertexing and event plane angle determination. Additionally, a forward vertex detector (FVTX) covering $1.0<|\eta|<3.0$ and composed of high-efficiency silicon mini-strips ${ }^{40}$ provides an independent event plane angle determination. A description of the PHENIX detector can be found in ref. ${ }^{41}$.

Event selection. A minimum bias (MB) interaction trigger is provided by the $\mathrm{BBC}$, which requires at least one hit tube in both the south $(\eta<0$, Au-going direction) and north $(\eta>0, \mathrm{p} / \mathrm{d}$-going direction), along with an online vertex within $\left|z_{\text {vertex }}\right|<10 \mathrm{~cm}$ of the nominal interaction region. In addition to the MB trigger, a high-multiplicity trigger requiring $>35(>40)$ hit tubes in the Au-going BBC provided a factor of 25 (188) enhancement of high-multiplicity events in p+ $\mathrm{Au}(\mathrm{d}+\mathrm{Au})$ collisions. A more precise offline collision vertex is determined using timing information in the BBC and is constrained to $\left|z_{\text {vertex }}\right|<10 \mathrm{~cm}$ in order to be sufficiently inside the acceptance of the detector. Events containing more than one nucleus-nucleus collision, referred to as double interaction events, are rejected using an algorithm based on BBC charge and timing information described in ref. ${ }^{24}$. Event centrality is determined using the total charge collected in the south $\mathrm{BBC}$, as described in ref. ${ }^{28}$. We require an event centrality of $0-5 \%$ to select events with the highest multiplicity, where the signal of interest is strongest. In total, 322 (636) million $\mathrm{p}+\mathrm{Au}(\mathrm{d}+\mathrm{Au})$ events are analysed.

Track selection. Quality cuts are applied to reconstructed particle tracks requiring hits in both the DC and the outermost PC layer with a required $3 \sigma$ level of agreement. This removes the majority of tracks that do not originate from the primary collision. Further details can be found in refs ${ }^{23-25}$.

Event plane determination. The third-order symmetry plane angle, $\psi_{3}$, is measured using the south BBC via the standard method ${ }^{42}$. Namely

$$
\psi_{3}=\frac{1}{3} \arctan \frac{\sum_{i}^{N} \sin 3 \phi_{i}}{\sum_{i}^{N} \cos 3 \phi_{i}}
$$

where $N$ is the number of particles and $\phi_{i}$ is the azimuthal angle of each particle. The $\psi_{3}$ resolution, $R\left(\psi_{3}\right)$, is calculated using the three-subevent method that correlates measurements in the south BBC, south FVTX and central arms. The calculated resolutions are $6.7 \%$ and $5.7 \%$ in $\mathrm{p}+\mathrm{Au}$ and $\mathrm{d}+\mathrm{Au}$ collisions, respectively.

Determination of $v_{3}$. The $v_{3}$ values are measured using the event plane method $^{26,42}$ as

$$
v_{3}=\frac{\left\langle\cos \left(3\left(\phi-\psi_{3}\right)\right)\right\rangle}{R\left(\psi_{3}\right)}
$$

where $\phi$ is the azimuthal angle of particles emitted at midrapitiy, $|\eta|<0.35$.

Systematic uncertainties. The systematic uncertainties reported are estimated according to the following methods for the measurements of $v_{3}$ in both $\mathrm{p}+\mathrm{Au}$ and $\mathrm{d}+\mathrm{Au}$ collisions.

The effect of remaining background tracks due primarily to photon conversions and weak decays is estimated by comparing the $v_{3}$ values when requiring a tighter matching between the track projection and hits in PC3. We find that this changes the $v_{3}$ by $\pm 4 \%$ and $\pm 7 \%$ in $\mathrm{p}+\mathrm{Au}$ and $\mathrm{d}+\mathrm{Au}$ collisions, respectively, independent of $p_{\mathrm{T}}$

The effect of double interaction event selection is estimated by comparing the $v_{3}$ values when requiring a tighter cut on the rejection. This yields a change in the $v_{3}$ of $\pm 3 \%$ a nd $\pm 2 \%$ in $\mathrm{p}+\mathrm{Au}$ and $\mathrm{d}+\mathrm{Au}$ collisions, respectively, independent of $p_{\mathrm{T}}$.

Uncertainty in the event plane resolution comes from two sources. The first is the statistical uncertainty inherent in the resolution calculation, which yields a $\pm 13 \%$ and $\pm 17 \%$ uncertainty in $p+$ Au and $d+$ Au collisions, respectively. Additionally, the resolution is calculated using central arm tracks over two different $p_{\mathrm{T}}$ regions. This leads to an uncertainty of $\pm 7 \%$ and $\pm 34 \%$ in $\mathrm{p}+\mathrm{Au}$ and $\mathrm{d}+\mathrm{Au}$ collisions, respectively.

We also include an uncertainty due to the choice of event plane detector. In p+ Au collisions, this is determined by comparing the $v_{3}$ calculated using event planes determined by the south BBC and FVTX and is found to be $<1 \%$. We find that the results are consistent within uncertainties, as expected. In $\mathrm{d}+\mathrm{Au}$ collisions, $v_{3}$ is also calculated using an alternative method utilizing two-particle correlations. Based on a ratio of the $v_{3}$ values calculated using the two-particle correlation and event plane methods, we assign a $\pm 17 \%$ systematic uncertainty.

In $v_{3}$, non-flow decreases the amplitude of the measured signal ${ }^{25}$, and its contribution increases with increasing $p_{\mathrm{T}}$. To estimate the non-flow contribution we calculate a normalized correlation function between midrapidity tracks and BBC photomultiplier (PMT) tubes

$$
\begin{gathered}
S\left(\Delta \phi, p_{\mathrm{T}}\right)=\frac{\mathrm{d}\left(Q_{\mathrm{PMT}} N_{\text {sameevent }}^{\mathrm{track}\left(p_{\mathrm{T}}\right)-\mathrm{PMT}}\right)}{\mathrm{d} \Delta \phi} \\
C\left(\Delta \phi, p_{\mathrm{T}}\right)=\frac{S\left(\Delta \phi, p_{\mathrm{T}}\right)}{M\left(\Delta \phi, p_{\mathrm{T}}\right)} \frac{\int_{0}^{2 \pi} M\left(\Delta \phi, p_{\mathrm{T}}\right)}{\int_{0}^{2 \pi} S\left(\Delta \phi, p_{\mathrm{T}}\right)}
\end{gathered}
$$

where $Q_{\mathrm{PMT}}$ is the charge on the PMT in the pair and $N_{\text {sameevent }}^{\operatorname{track}\left(p_{T}\right)-\mathrm{PMT}}$ is the number of track-PMT pairs from the same event. $M\left(\Delta \phi, p_{\mathrm{T}}\right)$ is determined in the same way as $S\left(\Delta \phi, p_{\mathrm{T}}\right)$ but with one particle in one event and another particle in a different event (the so-called mixed event technique). This normalization procedure accounts for acceptance effects and produces a correlation function of order unity. Next, we fit $C\left(\Delta \phi, p_{\mathrm{T}}\right)$ with a Fourier expansion:

$$
C(\Delta \phi)=1+\sum 2 c_{n}\left(p_{\mathrm{T}}\right) \cos (n \Delta \phi)
$$

We do this process for both systems in which we want to estimate the non-flow $(\mathrm{p}+\mathrm{Au}$ or $\mathrm{d}+\mathrm{Au})$ and for $\mathrm{p}+\mathrm{p}$ at the same collision energy. We use the Fourier coefficients $c_{n}$ to find the non-flow contribution to the $v_{n}$ values in a given system

$$
\text { nonflow ratio }=\frac{c_{n}^{\mathrm{p}+\mathrm{p}}\left(p_{\mathrm{T}}\right)}{c_{n}^{\text {system }}\left(p_{\mathrm{T}}\right)} \frac{\left\langle Q^{\mathrm{p}+\mathrm{p}}\right\rangle}{\left\langle Q^{\text {system }}\right\rangle}
$$

where $\langle Q\rangle$ is the average BBC charge for the system. The ratio of average charges normalizes the $c_{n}$ by multiplicity. The assumption is that $c_{n}^{p+p}$ is entirely due to non-flow such that the deviation of the ratio from one is taken as an estimate of the non-flow. It is included as a $p_{\mathrm{T}}$-dependent systematic uncertainty that decreases the $\mathrm{p}+\mathrm{Au}(\mathrm{d}+\mathrm{Au}) v_{3}$ by $21 \% \rightarrow 114 \%(18 \% \rightarrow 27 \%)$

An explanation of the ${ }^{3} \mathrm{He}+\mathrm{Au}$ uncertainties can be found in ref. ${ }^{25}$.

Comparison of theory to data. The level of agreement between the different theoretical calculations and the data presented in this work is quantified by performing a least-squares fit incorporating a careful treatment of various types of systematic uncertainties, following ref. ${ }^{31}$.

The non-flow uncertainty is the dominant source of systematic uncertainty in all six measurements. It is known to be point-to-point correlated as a function of $p_{\mathrm{T}}$, to contribute asymmetrically, and to be anti-correlated between $v_{2}$ and $v_{3}$. Namely, the non-flow can only reduce the measured $v_{2}$ while simultaneously only increasing the $v_{3}$.

All remaining measurement uncertainties are assumed to be uncorrelated between $v_{2}$ and $v_{3}$ and are assumed to contribute in the following ways: (1) as point-to-point uncorrelated uncertainties; (2) as point-to-point anti-correlated uncertainties (for example a tilt in the $p_{\mathrm{T}}$ dependence); and (3) as point-to-point correlated uncertainties.

The total systematic uncertainty (excluding the non-flow) is taken to contribute a fraction of its value to each of the above types. A conservative approach is taken, and these fractions are allowed to vary independently for each measurement within reasonable limits.

The bands around the theoretical calculations shown in Fig. 3 indicate some subset of theoretical uncertainties that differs between the models. We make the assumption that the dominant contribution is a point-to-point correlated uncertainty that is additionally correlated between $v_{2}$ and $v_{3}$. Given their small uncertainties, the inclusion of this treatment has little effect on the results for either SONIC or MSTV. It has the largest effect with iEBE-VISHNU, however its inclusion does not affect the relative ordering of the agreement discussed below. The AMPT calculations are taken from ref. ${ }^{35}$, which calculates $v_{2}$ and $v_{3}$ relative to the initial participant nucleon plane, utilizing the so-called 'string melting' mechanism, and a parton interaction cross-section of $\sigma=1.5 \mathrm{mb}$.

We calculate a $p$ value from the least-squares minimization in the standard way, where the number of degrees of freedom is simply the total number of data points, as there are no free parameters in the comparison. SONIC provides a very good description of the data with respective $p$ values of $0.97,0.69,0.47 \mathrm{in} \mathrm{p} / \mathrm{d} /{ }^{3} \mathrm{He}+$ $\mathrm{Au}$ collisions individually, and a combined $p$ value of 0.90 . The close to unity combined value may indicate a modest overestimate of the statistical or systematic uncertainties. iEBE-VISHNU yields worse $p$ values of $0.09,0.31,0.43$ for $\mathrm{p} / d /{ }^{3} \mathrm{He}+$ $\mathrm{Au}$, respectively, and a combined $p$ value of 0.14 . The larger $p$ value for SONIC compared with iEBE-VISHNU is driven by the non-flow uncertainty. The fact that 
SONIC tends to underpredict the $v_{2}$ while overpredicting the $v_{3}$ is mitigated by the non-flow uncertainty, while iEBE-VISHNU's worse description of the $p_{\mathrm{T}}$ dependence in $\mathrm{p} / \mathrm{d}+\mathrm{Au}$ is not compensated for by the relatively small remaining uncertainty. Both MSTV and AMPT yield a very poor description of the data with total $p$ values of $9.8 \times 10^{-17}$ and $2.7 \times 10^{-43}$, respectively. MSTV is particularly hindered by its $\mathrm{p}+\mathrm{Au}$ calculation that has a $p$ value of $7.07 \times 10^{-17}$ compared with $\mathrm{d}+\mathrm{Au}$ and ${ }^{3} \mathrm{He}+\mathrm{Au}$ that have $p$ values of 0.011 and 0.007 , respectively. AMPT yields extremely poor results for all three systems, the largest of which is on the order of $10^{-7}$.

\section{Data availability}

All raw data for this study were collected using the PHENIX detector at

Brookhaven National Laboratory. Data tables for the results reported in this paper and other findings of this study are publicly available on the PHENIX website (https://www.phenix.bnl.gov/phenix/WWW/info/data/ppg216_data.html) or from the corresponding author upon reasonable request.

\section{References}

39. Adcox, K. et al. PHENIX central arm tracking detectors. Nucl. Instrum. Meth. A 499, 489-507 (2003).

40. Aidala, C. et al. The PHENIX forward silicon vertex detector. Nucl. Instrum. Meth. A 755, 44-61 (2014).

41. Adcox, K. et al. PHENIX detector overview. Nucl. Instrum. Meth. A 499, 469-479 (2003).

42. Poskanzer, A. M. \& Voloshin, S. A. Methods for analyzing anisotropic flow in relativistic nuclear collisions. Phys. Rev. C 58, 1671-1678 (1998). 